\title{
Comparison of Gutinase Separation in Different Chromatographic Media
}

\author{
Abdul Wahab Mohammad *,1 \\ Jamaliah Md.Jahim ${ }^{1}$ \\ Suhaila Johar ${ }^{1}$ \\ Osman Hassan ${ }^{2}$ \\ ${ }^{1}$ Department of Chemical \& Process Engineering, Faculty of Engineering \& Built Environment, \\ Universiti Kebangsaan Malaysia, 43600 Bangi, Selangor. \\ ${ }^{2}$ School of Chemical Sciences and Food Technology, Faculty of Science and Technology, \\ Universiti Kebangsaan Malaysia, 43600 Bangi, Selangor. \\ *e-mail : wahabm@ukm.eng.my
}

Cutinase is a hydrolytic enzyme that has both properties of lipase and esterase, thus finding its use in many areas. Previous studies have investigated both upstream and downstream processes for cutinase production from microbial source. However, no study has yet to address the use of membrane chromatography for cutinase purification, which is more favourable in terms of process resolution and product throughput as compared to the conventional gel chromatography. Hydrophobic interaction was chosen as the separation mechanism for cutinase purification in this study. The optimisation of cutinase purification in two different types of chromatographic media; conventional packed-gel and membrane matrix, were pre-determined by the best compromise between the recovery and purity of the purified cutinase. It was found that the optimised condition were of $\mathrm{pH} 4.0$ and $1.0 \mathrm{M}$ ammonium sulfate for the conventional column ( $50 \%$ recovery, 4.8-fold purity) and $\mathrm{pH} 6.0$ with $1.5 \mathrm{M}$ ammonium sulfate for the membrane-matrix column ( $87 \%$ recovery, 30 -fold purity). Preferential interaction analysis was used to describe the protein chromatographic behaviour in each chromatographic media. Graph of natural algorithm of protein retention data to the function of salt concentration at $\mathrm{pH}$ 4.0 and 6.0 for each column were plotted. It was found that at the optimum pH condition for gel-packed column, a small amount of ammonium sulfate was sufficient to achieve maximum cutinase recovery and purity since the effect of salt at that particular $\mathrm{pH}$ were less significant. Consequently, the number of released water molecules were calculated and it was observed that for membrane column, larger number of water was released at $\mathrm{pH} 6.0$ illustrating more protein were bounded to the stationary phase, thus explaining the optimum $\mathrm{pH}$ condition of the particular column.

Keywords: Purification, Cutinase, Packed-bed, Membrane matrix, Hydrophobic interaction, Preferential interaction analysis 


\section{INTRODUCTION}

Cutinase (EC 3.1.1.74) is a cutindegrading enzyme which originates from phytophatogenic fungi (Carvalho et al. 1998). Fungal cutinase is mostly consist of single peptide having molecular weight of 22-26 kDa in range (Kolattukudy et al. 1981). Cutinase have double properties of an esterase and lipase, hence it has been applied in many area such as detergent and personal care products, oleochemicals and dairy industries (Dutta et al. 2009). Such wide application has led to numerous studies of cutinase production and purification. Crude cutinase from the fermentation process has been purified using many separation techniques such as expended bed adsorption (EBA), affinity chromatography and also hydrophobic interaction chromatography (HIC) (Wang et al. 2000, Nilsson et al. 2003, Kepka et al. 2005, Lienqueo et al. 2008). However, cutinase has yet to be purified using hydrophobic interaction membrane chromatography (HIMC) which has favorable advantage in mass transport properties as compared to the conventional packed-bed HIC (Ghosh 2001, Yu et al. 2008).

$\mathrm{HIC}$ has gained a lot of attention as the choice of chromatographic separation as it is able to produce equally efficient separation at relatively low cost and more benign separation (Ghosh and Wang 2006). However, there are some restriction in solutes transport in the packed-bed chromatography which complicates the scale up of such process (Yu et al. 2008). Recent studies have developed an alternative media for chromatographic process which utilises the advantages of membrane technology in overcoming the problem experienced in the conventional packed-bed column (Ghosh and Wong 2006, Huang et al. 2009, Pereira et al. 2010).

Perkins et al. in 1997 has expended the preferential interaction theory (PIT) to describe the salt effect in HIC. This theory used the same approach as solvophobic theory which examines the effect of salts on the properties of the solvent with the advantage of direct physical meaning of the parameters as compared to the lumped factors in the solvophobic theory. Theoretically, during binding of protein to the hydrophobic ligand, water around the protein-ligand complex will redistribute which results in a decrease in hydrophobic exposed area, thus water is released (Xia et al. 2004). Therefore, the analysis could be further discussed by analysing the number of released water molecules of the respective solutes and stationary phase applied.

This study will focus on the separation behaviour in different chromatographic media used for the purification of cutinase. A mixture of cutinase and bovine serum albumin (BSA) was prepared to act as the model protein in simulating the purification process. The cutinase purification process was initially determined by optimising the mobile phase $\mathrm{pH}$ and the kosmotropic salt concentration condition to achieve the maximum cutinase recovery and purity possible. Ammonium sulfate was chosen as the kosmotropic salt used to enhance the binding of protein to the stationary phase. Isocratic elution experiments were conducted to obtain the protein retention data which were eventually used in 
Recovery $=\frac{\text { Total activity }(\mathrm{U}) \text { after HIMC }}{\text { Total activity (U)in feed solution }} \times 100$

Purification factor $=\frac{\text { Specific activity }\left(\mathrm{Umg}^{-1}\right) \text { after HIMC }}{\text { Specific activity }\left(\mathrm{Umg}^{-1}\right) \text { in feed solution }}$

determining the preferential interaction parameters. The resulting parameters were then utilised to interpret the chromatographic behaviour of protein in each media.

\section{THEORY AND EXPERIMENTAL PROCEDURES}

\section{Calculation for recovery and purity}

The performance of the chromatographic process were evaluated by the ability of the process to produce high recovery and purity of cutinase. The recovery and purity of cutinase were calculated using Eq.(1) and Eq.(2)

\section{Preferential interaction theory of HIC}

The PIT model for HIC (Xia et al. 2004, Lienqueo et al. 2007) were used to describe the correlation between solute's capacity factor and salt concentration. The relationship is expressed as follows:

$$
\ln \left(k^{\prime}\right)=\alpha+\beta m_{3}+\gamma \ln \left(m_{3}\right)
$$

where $k^{\prime}$ is the capacity or rentention factor which can be determined using Eq. (4), $\alpha$ is a constant and $m_{3}$ refers to the molality of the salt.

$$
k^{\prime}=\frac{t_{r}-t_{0}}{t_{0}}
$$

The terms $t_{r}$ and $t_{0}$ in Eq. (4) are referred to the retention time of the adsorbed protein and the unretained solutes, respectively. The $\beta$ and $\gamma$ are the preferential interaction parameters to calculate the total number of water molecules and salt ions released during binding process by using the following equation (Perkins et al. 1997):

$$
-\left(\Delta v_{1}\right)=\frac{\beta \mathrm{g} m_{1}}{n}
$$

where $m_{1}$ is molality of water, $n$ is valence of salt ions and $g=\left(\partial \ln \left(m_{3}\right) /(\partial \ln (a \pm)), a\right.$ is the activity of ions and $-(\Delta v 1)$ is the number of water molecules released during binding process. The value of $g$ can be computed using the Debye-Hückel equation.

\section{Preparation of buffer solutions}

All buffers used for the chromatographic process were prepared according to the respective $\mathrm{pH}$ range; sodium acetate for $\mathrm{pH}$ 4.0 and sodium phosphate for $\mathrm{pH} \mathrm{6.0,} \mathrm{all}$ fixed to $50 \mathrm{mM}$ of concentration. Two sets of buffers were used for each run, one in presence of ammonium sulfate in range of 0.8-2.2 M; known as the binding buffer, and another without the presence of salting-out salt which act as the elution buffer. All buffers were filtered and degassed prior use. 
Table 1. Specification of HIC and HIMC column

\begin{tabular}{ccc}
\hline & HIC & HIMC \\
\hline Media & Sepharose & Stabilised and reinforced cellulose \\
Ligand & Butyl & Phenyl \\
Media size $(\mu \mathrm{m})$ & $90^{\star}$ & $>3^{* *}$ \\
Column volume $(\mathrm{ml})$ & 1.0 & 3.0 \\
\hline
\end{tabular}

Note: ${ }^{*}$ mean bead size ${ }^{* *}$ nominal pore size

\section{Sample preparation}

The protein samples for the purification experiment, comprising of cutinase (Novozyme 51032) and BSA, were prepared in binding buffer to simulate the separation of protein for the purification process. For isocratic elution experiment, similar approach was used to prepare the samples but only to contain single protein of cutinase. All samples were centrifuged at 10,000 rpm for 20 minutes before injecting samples to the chromatography columns.

\section{Chromatographic runs}

All the chromatographic experiments were conducted on AKTAprime FPLC system. Flow rate was maintain at $1.0 \mathrm{ml}$ $\min -1$ throughout the process. Two types of column were tested; Butyl FF for HIC column and Sartobind Phebyl nano for HIMC column. The specification of both columns were summarised in Table 1. Purification of cutinase from the protein mixture were performed on both columns by employing gradient elution. The protein isocratic retention data were obtained by performing isocratic elution on each columns. Binding buffer which contained ammonium sulfate were equilibrated for about 5 column volumes (CV) then followed by injection of $100 \mu$ of samples. The obtained retention data were then recorded and process were repeated to obtain duplicate data.

\section{Protein and cutinase activity assay}

Bradford asssay (Bradford 1976) and cutinase activity assay (Kumar et al. 2005) were conducted to determine protein content and cutinase activity in the crude and purified sample. These data were then used to calculate the cutinase recovery and purity.

\section{Parameter regression}

The isocratic retention data were plotted and Eq. (3) was fitted using Matlab R2008b software to obtain the values of each parameters. Parameter regression was performed to minimised the sum of squared residuals (SSR) between experimental data and the model response.

\section{RESULTS AND DISCUSSION}

\section{Purification of cutinase}

Purification of cutinase were performed on various buffer $\mathrm{pH}$ and ammonium sulfate concentration. The eluted protein were collected in fractions and checked for its protein concentration and cutinase activity. The recovery and purity of the purified cutinase were quantified. It was observed that the best compromise of 

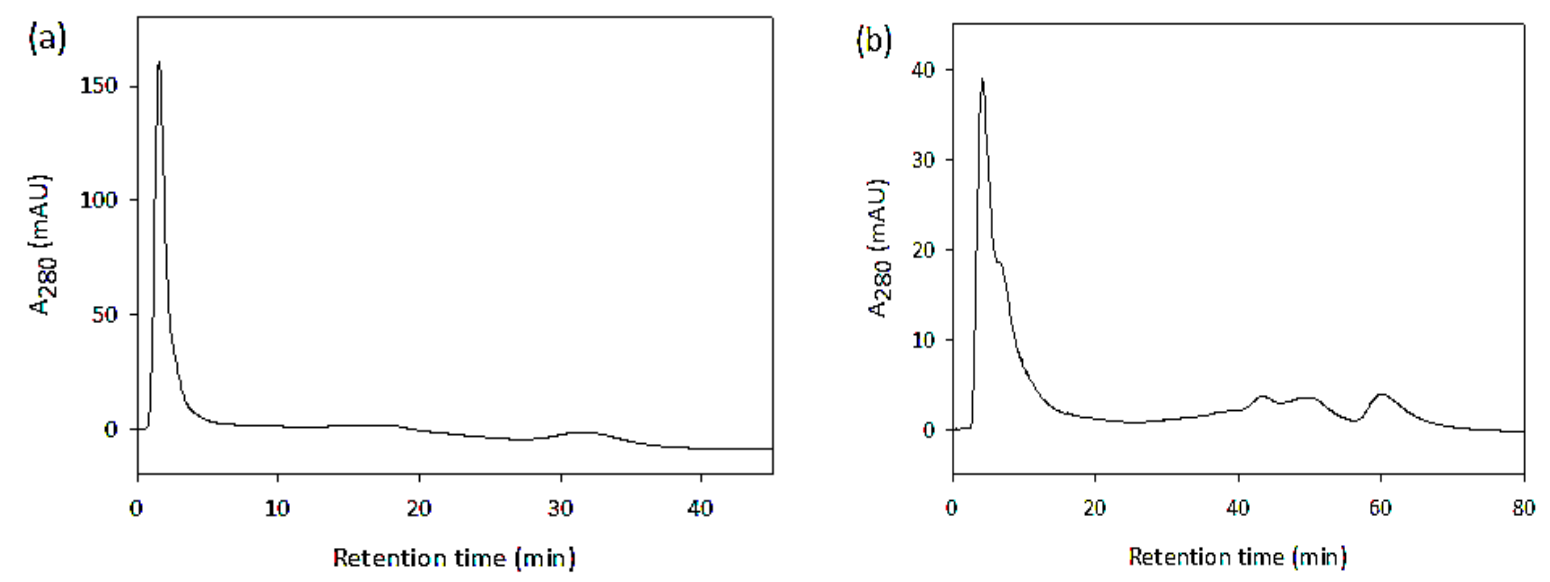

Fig. 1: Chromatograms of the optimised condition for (a) HIC and (b) HIMC columns
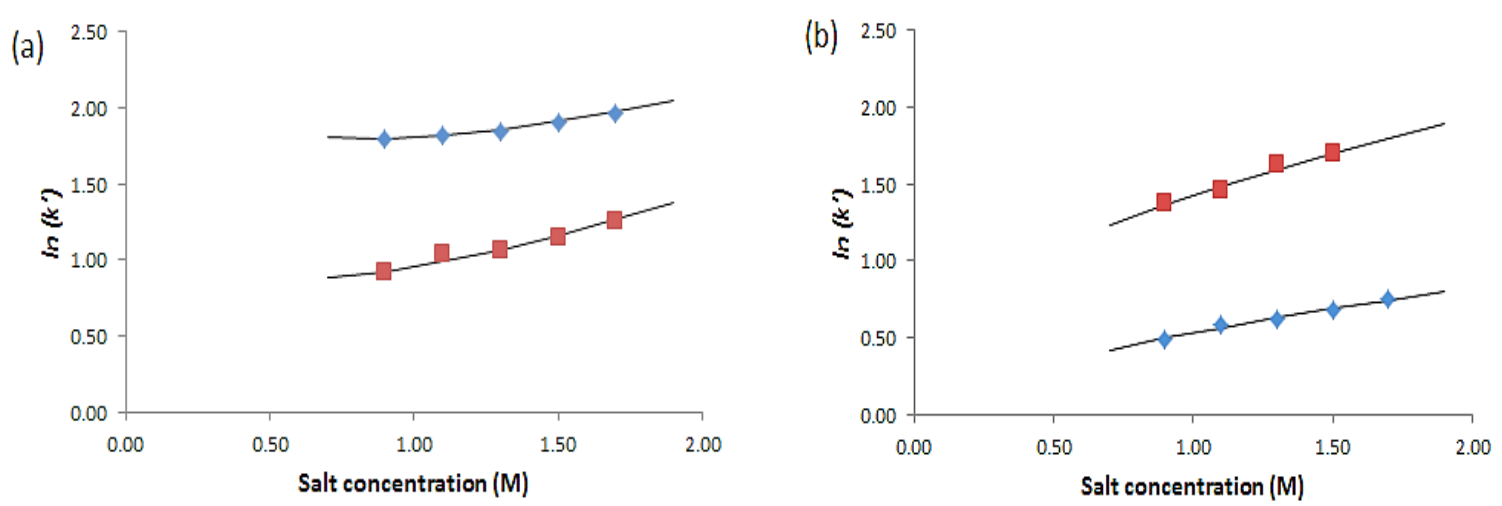

Fig. 2: Plots of natural algorithm of protein retention data against ammonium sulfate concentration at pH 4.0 and 6.0 for (a) HIC and (b) HIMC columns. ( $\diamond), \mathrm{pH} 4.0$; ( $\square$ ), pH 6.0.

recovery and purity obtained for $\mathrm{HIC}$ was at $\mathrm{pH} 4.0$ and $1.0 \mathrm{M}$ ammonium sulfate. Meanwhile for HIMC column, pH 6.0 and $1.5 \mathrm{M}$ salt concentration was found to be the optimum condition for the maximum output of cutinase recovery and purity. Table 2 shows the result summary of the optimum condition for cutinase purification for each type of column.

Table 2. Summary of cutinase purification result

\begin{tabular}{lcc}
\hline & HIC & HIMC \\
\hline Recovery (\%) & 50 & 87 \\
Purity (fold) & 4.8 & 30 \\
\hline
\end{tabular}

Figure 1 shows the chromatogram of the optimised condition for HIC and HIMC column. Cutinase was eluted at 32 and 60 minutes at retention time, respectively. It can also be observed that the peak in HIC is rather broad as compared to the sharp peak produced by the HIMC column.

\section{Protein retention factor data}

PIT was employed to describe the protein behaviour in different chromatographic condition. Isocratic elution experiment were conducted to obtain the capacity factors data for each type of columns at various $\mathrm{pH}$ and ammonium sulfate concentration. The protein retention factors data ( $\ln k$ ) were plotted against salt concentration in range 
of $0.9-1.7$ M. Generally, the capacity factors would increase with increasing of kosmotropic salt concentration (Xia et al. 2004). A high value of In k' would indicate that the hydrophobic interaction was strong due to the increasing time needed to desorp the protein from the adsorber.

Figure 2 shows the plots of natural algorithm of the retention factors versus the ammonium sulfate molal concentration at $\mathrm{pH} 4.0$ and 6.0 for both columns. From this plot, it was observed that the optimum $\mathrm{pH}$ of each column has higher $\ln k^{\prime}$ values compared to the other $\mathrm{pH}$. It can also be seen that at $\mathrm{pH} 4.0$ of HIC column, the effect of salt concentration to $\ln k^{\prime}$ were less obvious than in $\mathrm{pH}$ 6.0. Therefore, low salt concentration were sufficient to obtain maximum recovery and purity of cutinase.

\section{Determination of preferential interaction parameters}

The plots as in Figure 2 was fitted with Eq. (3) to obtain the values of the preferential interaction parameters. These values could later be used to evaluate the effects of salt, $\mathrm{pH}$ and stationary phase (Lienqueo et al. 2007) of the particular purification process. The values are tabulated in Table 3.

Table 3. Values of the preferential interaction parameters

\begin{tabular}{ccccc}
\hline Column & \multicolumn{2}{c}{ HIC } & \multicolumn{2}{c}{ HIMC } \\
\hline $\mathbf{p H}$ & $\mathbf{4 . 0}$ & $\mathbf{6 . 0}$ & $\mathbf{4 . 0}$ & $\mathbf{6 . 0}$ \\
$\boldsymbol{\alpha}$ & 1.094 & 0.098 & 0.346 & 1.079 \\
$\boldsymbol{\beta}$ & 0.711 & 0.853 & 0.185 & 0.345 \\
$\boldsymbol{\gamma}$ & -0.628 & -0.535 & 0.157 & 0.253 \\
\hline
\end{tabular}

From Table 3, both columns for each $\mathrm{pH}$ recorded positive values of $\beta$, which shows that the protein retention increases with increasing salt concentration (Chen et al. 2008). The significance of the $\beta$ parameter will be described in the later section.

\section{Water release values}

The total number of released water andsalt ions molecules were calculated based on Eq. (5) using the $\beta$ values determined in section Protein retention factor data. A high number of water molecules released during binding process demonstrate that the total wetted area has decreased due to the binding of hydrophobic patches on protein surface to the hydrophobic ligands (Perkins et al. 1997). Thus stronger bind should show higher number of water release value. Table 4 summarised the number of released water molecules at $\mathrm{pH} 4.0$ and 6.0 of the HIC and HIMC column. Sartobind Phenyl nano column has relatively low number of released molecules as compared to the Butyl FF. A study done by To and Lenhoff (2007) found that the number released values are statistically independent of protein retention strength and also on the adsorbent and protein properties. It is also not sensitive enough to allow prediction of adsorption selectivity. Nonetheless, the result shows that number of water released increased as increasing $\mathrm{pH}$ for both columns. For HIMC column, protein binding is higher at $\mathrm{pH}$ 6.0, which explains the better purification result at that particular $\mathrm{pH}$. For HIC column, although the $-(\Delta v 1)$ value is slightly higher at $\mathrm{pH} 6.0$, but since the $\ln \left(k^{\prime}\right)$ range is higher at $\mathrm{pH} 4.0$; which indicates better separation, therefore $\mathrm{pH} 4.0$ is the best chromatographic condition. 
Table 4. Water released values at $\mathrm{pH} 4.0$ and 6.0 of HIC and HIMC columns

\begin{tabular}{ccccc}
\hline Column & \multicolumn{2}{c}{ HIC } & \multicolumn{2}{c}{ HIMC } \\
\hline $\mathbf{p H}$ & $\mathbf{4 . 0}$ & $\mathbf{6 . 0}$ & $\mathbf{4 . 0}$ & $\mathbf{6 . 0}$ \\
$-\left(\Delta v_{1}\right)$ & 21 & 25 & 5 & 10 \\
\hline
\end{tabular}

\section{CONCLUSION}

It was observed that for each chromatographic media, different optimum condition were recorded in achieving maximised recovery and purity of cutinase. The recovery and purity obtained by HIC column is much lower from the HIMC column. Such behaviour is observed by using the PIT to evaluate the effect of $\mathrm{pH}$, salt concentration and the stationary phase. The plots of natural algorithm of the protein retention data versus the ammonium sulfate concentration all increases with increasing salt concentration. At pH 4.0 for HIC column, the effect of salt concentration was less obvious, thus only small amount of ammonium sulfate in necessary to promote separation. It can also be observed that higher protein binding had occurred at $\mathrm{pH}$ 6.0 which correlates with the optimum $\mathrm{pH}$ condition for the HIMC column.

\section{ACKNOWLEDGEMENT}

Highest gratitute is expressed to Universiti Kebangsaan Malaysia for providing the funds to conduct this research works through the UKM-AP-BPB12-2009 university grant.

\section{REFERENCES}

1. Bradford, M. M. (1976). A rapid and sensitive method for the quantitation of microgram quantities of protein utilizing the principle of protein-dye binding, Anal. Biochem., 72, 248-254.

2. Carvalho, C. M. L., Aires-Barros, M. R., Cabral, J. M. S. (1998). Cutinase structure, function and biocatalytic applications, Electron. J. Biotechnol., 1, 160-173.

3. Chen, J., Yang, T., Cramer, S. M. (2008). Prediction of protein retention times in gradient hydrophobic interaction chromatographic systems, J. Chromatogr., A, 1177, 207-214.

4. Dutta, K., Sen, S., Veeranki, V. D. (2009). Production, characterization and applications of microbial cutinases, Process Biochem., 44, 127-134.

5. Ghosh, R. (2001). Separation of proteins using hydrophobic interaction membrane chromatography, J. Chromatogr., A, 923, 59-64.

6. Ghosh, R., and Wang, L. (2006). Purification of humanized monoclonal antibody by hydrophobic interaction membrane chromatography, J. Chromatogr., A, 1107, 104-109.

7. Ghosh, R., and Wong, T. (2006). Effect of module design on the efficiency of membrane chromatographic separation processes, J. Membr. Sci., 281, 532-540.

8. Huang, R., Mah, K. Z., Malta, M., Kostanski, L. K., Filipe, C. D. M., Ghosh, 
R. (2009). Chromatogrphic separation of proteins using hydrophobic membrane shielded with an environment-responsive hydrogel, J. Membr. Sci., 345, 177-182.

9. Kepka, C., Colleet, E., Roos, F., Tjerneld, F., Veide, A. (2005). Two-step recovery process for tryptophan tagged cutinase: Interfacing aqueous twophase extracton and hydrophobic interaction chromatography, J. Chromatogr., A, 1075, 33-41.

10. Kolattukudy, P.E., Purdy, R.E., Maiti, I.B. (1981). Cutinases from fungi and pollen, Methods Enzymol., 71, 652-664.

11. Kumar, S., Kinon, K., Upadhyay, A., Kanwar, S. S., Gupta, R. (2005). Production, purification, and characterization of lipase from thermophilic and alkaliphilic Bacillus coagulans BTS-3, Protein Expression Purif., 41, 38-44.

12. Lienqueo, M. E., Mahn, A., Salgado, J.C., Asenjo, J. A. (2007). Current insights on protein behaviour in hydrophobic interaction chromatography, J. Chromatogr., B, 849, 53-68.

13. Lienqueo, M. E., Salazar, O., Calado, C. R. C., Fonseca, L. P., Cabral, J. M. S. (2008). Influence of tryptophan tags on the purification of cutinase, secreted by recombinant Saccharomyces cerevisiae, using cationic expended bed adsorption and hydrophobic interaction chromatography, Biotechnol. Lett., 30, 1353-1358.

14. Nilsson, A., Neves-petersen, M. T., Johansson, H.-O., Jansson, J., Schillén, K., Tjerneld, F., Petersen, S. B. (2003). Tryptophan-tagged cutinase studied by steady state fluorescence for understanding of tag interactions in aqueous two-phase systems, Biochim. Biophys. Acta, 1646, 57-66.

15. Pereira, L. R., Prazeres, D. M. F., Mateus, M. (2010). Hydrophobic interacton membrane chromatography for plasmid DNA purification: Design and optimization, J. Sep. Sci., 33, 1175-1184.

16. Perkins, T. W., Mak, D. S., Root, T. W., Lightfoot, E. N. (1997). Protein retention in hydrophobic interaction chromatography: modelling variation with buffer ionic strength and column hydrophobicity, J. Chromatogr., A, 766, 1-14.

17. To, B. C. S., and Lenhoff, A. M. (2007). Hydrophobic interaction chromatography of proteins I. The effects of protein and adsorbents properties on retention and recovery, $J$. Chromatogr., A, 1141, 191-205.

18. Wang, G.-Y., Michailides, T. J., Hammock, B. D., Lee, Y.-M., Bostock, R. M. (2000). Affinity purification and characterization of a cutinase from the fungal plant pathogen Monilinia fructicola (Wint.) honey, Arch. Biochem. Biophys., 382, 31-38.

19. Xia, F., Nagrath, D., Garde, S., Cramer, S. M. (2004). Evaluation of selectivity changes in HIC systems using a preferential interaction based analysis. Biotechnol. Bioeng., 87, 354-363.

20. Yu, D., McLean, M. D., Hall, J. C., Ghosh, R. (2008). Purification of monoclonal antibody from tobacco extract using membrane-based bioseparation techniques, J. Membr. Sci., 323, 159-166. 